\title{
Interleukin 2
}

\author{
Side effects are acceptable
}

Immunotherapy has been a long time coming, but after many false starts a drug - interleukin 2-has emerged as a treatment capable of inducing complete responses in patients with cancers previously thought to be completely unresponsive.

In metastatic melanoma and renal cell carcinoma responses are seen in between a quarter and a third of patients, and already these responses have been reflected in improved survival. ${ }^{1}$ Not everyone, however, has welcomed the new drug. One famous cancer specialist complained, "Treatment with high dose interleukin 2 is an awesome experience. It requires weeks of hospitalisation, much of which must be spent in intensive care units if the patient is to survive the devastating toxic reactions ... it would not seem to merit further application in the compassionate management of patients with cancer." 2 This extreme view was reinforced by the Horizon television programme (Nova in the United States) that showed patients with cancer suffering from paroxysms of fever and bravely bearing cruel privations at the hands of medical experimenters, and all for little if any benefit ("Patients on trial," BBC2, 29 February 1988).

Oncologists are used to toxic drugs and to learning how to minimise this toxicity. Da Vita has recalled the early use of vincristine in childhood acute lymphoblastic leukaemia; a drug that once filled wards with paralysed children is now given to outpatients who go out and play afterwards. ${ }^{3}$ Oncologists have also found ways of using drugs that cause profound bone marrow suppression in such a way that the side effects experienced by the patient are tolerable.

Interleukin 2 is a novel kind of anticancer drug; most patients given it develop a new range of side effects, some of which may be unpleasant and most of which will be unfamiliar to oncologists. These are, however, insubstantial when compared with the effects of bone marrow transplantation or the standard platinum based chemotherapy for testicular teratoma. Moreover, the reported side effects generally relate to patients treated with the maximum tolerated dose of $24 \times 10^{6} \mathrm{IU} / \mathrm{m}^{2}$ (equivalent to $4 \times 10^{6}$ Cetus units $/ \mathrm{m}^{2}$ ) of interleukin 2 given by intravenous bolus eight hourly. ${ }^{+}$Side effects are much less severe when the drug is given by continuous infusion at a dose of $18 \times 10^{6} \mathrm{IU} / \mathrm{m}^{2}$ a day, as has been the rule in the European trials. ${ }^{5}$ With this regimen most patients may be treated in an ordinary oncology ward, and side effects are short lived and cease within a few hours of stopping the drug.

Most patients suffer influenza-like symptoms of malaise, fatigue, fever, anorexia, and myalgia, and these are more severe than are seen with treatment with interferon alfa. The patient usually prefers to be in bed. The symptoms may usually be controlled with paracetamol and occasionally with intravenous pethidine for rigors. Nausea and vomiting are much less severe than with cytotoxic drugs, but diarrhoea may be troublesome. Although anaemia, neutropenia, and thrombocytopenia are seen, they seldom merit any sort of supportive care. Mild hepatic dysfunction shows itself as transient rises in bilirubin concentrations and transaminase activities and prolongation of coagulation tests, but it generally causes no symptoms. Dry eyes and dry skin respond to simple treatment. A pruritic erythematous rash may occur; like that of graft versus host disease it may affect the palms and soles.

Of greater importance is the vascular leak syndrome. Although interleukin 2 has no direct effect on vascular endothelium, ${ }^{6}$ it stimulates the release of other cytokines, including interleukin 1, tumour necrosis factor, lymphocytotoxin, and interferon gamma, which when incubated with endothelial cells induce the appearance of activation antigens. ${ }^{7}$ Endothelial cell activation leads to the blood vessels becoming leaky to macromolecules. ${ }^{8}$

The movement of fluid from the vasculature into the tissues leads to hypovolaemia and hypotension and is responsible for gain in weight, peripheral oedema, dyspnoea, pulmonary oedema, and, in severe cases, neuropsychiatric effects such as disorientation, confusion, personality change, hallucinations, and drowsiness.

The normal response of the kidney to hypoperfusion is to increase secretion of renin and hence angiotensin II. This in turn results in the secretion of renal prostaglandins, which protect the kidney from ischaemic damage by causing dilatation of the renal vascular bed. Interleukin 2 has a direct inhibitory effect, however, on the secretion of renal prostaglandins, so that the normal response is blocked and the end results are ischaemia, oliguria, and prerenal renal failure. ${ }^{10}$ These effects may be made worse by the use of indomethacin in an attempt to control the influenza-like side effects, as this drug also inhibits synthesis of renal prostaglandins." Since dropping indomethacin from the protocol we and others have found the incidence of serious renal toxicity much reduced. ${ }^{12}$ And although prerenal renal failure has been a relatively common problem, acute tubular necrosis has not been reported. 
The management of the hypovolaemia and hypotension is based on giving colloid and the judicious use of pressor agents. Low dose dopamine infusions $(2-3 \mu \mathrm{g} / \mathrm{kg} / \mathrm{min})$ may be effective in maintaining the blood pressure but should be used with caution. Higher doses further decrease renal perfusion and have been associated with cardiotoxicity, including supraventricular tachycardias, ventricular ectopic beats, myocardial infarction, and cardiac arrest. ${ }^{13}$ One of the advantages of the treatment protocol of continuous infusion is that it gives scope for the infusion to be interrupted if the patient becomes hypotensive- which may be preferable to the use of pressor agents.

Two other unusual side effects have been noted: bowel perforation and hypothyroidism. In at least some patients the perforation was at the site of an unresected tumour, and histological examination has shown tumour necrosis. ${ }^{1+}$ Most of the patients who became hypothyroid had pre-existing antithyroid antibodies. ${ }^{15}$ It may be that the activation of natural killer cells provides effector cells for antibody dependent cellular cytotoxicity or that coexisting autoreactive $T$ cells have also been activated.

Despite this wide array of side effects in most patients they are predictable and may therefore be handled effectively. It is almost always possible for the patient to be treated in an ordinary hospital ward, and already reports have appeared of the successful use of interleukin 2 in outpatient departments. ${ }^{16}$ Fear of the side effects of anticancer drugs inhibits their use and drives patients who could benefit from them into the arms of alternative practitioners. Oncologists should learn how to use new drugs - even if the side effects are strange - so that their patients are not deprived of effective treatment.

Professor of Immunohaematology,

T J HAMBLIN

Royal Victoria Hospital,

Bournemouth NH1 4JG

Anonymous. Interleukin-2: sunrise for immunotherapy [Editorial]. Lancet 1989;i:308.

Moertel CG. On lymphokines, cytokines, and breakthroughs. FAMA 1986;256:3141.

Bloom M. Cancer MDs clash over interleukin therapy. Science 1987;235:154-5.

4 Rosenberg SA, Lotze MT, Muul LM, et al. Special report: observations on the systemic administration of autologous lymphokine activated killer cells and recombinant interleukin-2 to patients with metastatic cancer. N Engl F Med 1985;313:1485-92.

5 West WH, Taeur KW, Yanelli JR, et al. Constant infusion of recombinant interleukin-2 in adoptive immunotherapy of advanced cancer. $N$ Engl f Med 1987;316:898-905.

6 Kotasek D, Vercellotti GM, Ochoa AC, Bach FH, White JG, Jacob HS. Mechanism of cultured endothelial injury by lymphokine-activated killer cells. Cancer Res 1988;48:5528-32.

7 Cotran R, Pober JS, Gimbrone MA Jr, et al. Endothelial activation during interleukin-2 immunotherapy; a possible mechanism for the vascular leak syndrome. $\mathcal{F}$ Immunol 1987;139: 1883-8.

8 Cotran RS, Gimbrone MA Jr, Bevilacqua MP, Mendrick DL, Pober JS. Induction and detection of human endothelial activation antigen in vivo. 7 Exp Med 1986;164:661-6.

9 Denicoff KD, Rubinow DR, Papa MZ, et al. The neuropsychiatric effects of treatment with interleukin-2 and lymphokine-activated killer cells. Ann Intern Med 1987;107:293-300.
int

10 Christiansen NP, Skubitz KM, Nath K, Ochoa A, Kennedy BJ. Nephrotoxicity of continuous intravenous infusion of recombinant interleukin-2. Am F Med 1988;84:1072-5.

11 Dunn MJ. Non-steroidal anti-inflammatory drugs and renal function. Annu Rev Med 1984;35:411-28.

2 Sosman JA, Kohler PC, Hank JA, et al. Repetitive weekly cycles of interleukin-2. II. Clinical and immunological effects of dose schedule, and addition of indomethacin. $7 N C I$ 1988;80:1451-61.

13 Nora R, Abrams JS, Tait NS, Hiponia DJ, Silverman HJ. Myocardial toxic effects durin recombinant interleukin-2 therapy. $7 N C I$ 1989;81:59-63.

14 Schwartzentruber D, Lotze MT, Rosenberg SA. Colonic perforation: an unusual complication of therapy with high dose interleukin-2. Cancer 1988;62:2350-3.

15 Atkins MB, Mier JW, Parkinson DR, Gould JA, Berkman EM, Kaplan MM. Hypothyroidism after treatment with interleukin and lymphokine activated killer cells. $N$ Engl $\mathcal{J}$ Med 1988;318:1557-63.

16 Mitchell MS, Kempf RA, Harel W, et al. Effectiveness and tolerability of low dose cyclophosphamide and low dose intravenous interleukin-2 in disseminated melanoma. $9 \mathrm{Clin}$ Oncol phosphamide and $1988: 3: 409-24$.

\section{Advanced rectal cancer}

\section{New techniques are now available for conservative and palliative management}

The prognosis of advanced cancer of the rectum is poor; patients with liver metastases usually live no more than six to nine months. Despite this grim outlook many surgeons believe that abdominoperineal resection is good palliation for advanced rectal cancer - a belief often held because resection is the only method of palliation they know. Yet it cannot make sense to attempt resection in a patient with liver metastases and so condemn the patient to spending potentially the best three of his or her remaining six months convalescing after a major operation - and that is if all goes well. Furthermore, in elderly patients the risk to life of major resections is high. Surgeons should, therefore, look carefully at the audited results of their achievements with resection in palliating advanced rectal cancer. Those who have carried out such a close analysis have found the results disappointing and have sought alternative, less aggressive and dangerous methods of palliation.

In patients who are poor risks small potentially curable lesions can be managed successfully with local resection by the transanal' or transphincteric route. ${ }^{2}$ This approach is not usually feasible for advanced, large, or fixed tumours, and for these surgeons have used other non-radical approaches. Electrofulguration with electric cautery was reported in $1972^{3}$ and later modified and perfected. ${ }^{+}$Usually fulguration is performed through a large rigid endoscope, and this requires prolonged anal dilatation. The elderly patient may not tolerate this dilatation, which may have disastrous results on an already tenuous continence. Chemotherapy has been tried, and (as with so many advanced gastrointestinal cancers) the results have been cruelly disappointing with the drugs available. Intracavity local radiotherapy also requires wide stretching of the anus, ${ }^{5}$ and, though good results have been reported, ${ }^{6}$ it has not been widely adopted outside a few enthusiastic centres. Cryodestruction with liquid nitrogen is theoretically attractive, but despite initial enthusiasm few centres continue to use it. Furthermore, intercavity radiation and cryodestruction are suitable only for distal rectal tumours.

Two newer methods for local tissue destruction are now available. One is diathermy resection in a fluid mediumborrowing the techniques used by urologists for bladder and prostatic resection. In 1979 Zinken and colleagues described the use of a urological resectoscope in six patients with carcinoma of the lower rectum. ${ }^{7}$ The resectoscope was used to cut a hole and so to increase the size of the lumen and prevent obstruction during the limited life expectancy of patients with terminal disease. Since then many small series have been reported. Recent favourable reports place this technique in the forefront of non-operative debulking palliative manoeuvres. ${ }^{8}$ It possibly may be the procedure of choice in palliation of rectal tumours in patients with a limited prognosis because of liver metastases or advanced local node disease.

In other parts of the world the Nd-YAG laser is being employed to achieve similar ends and with similar results. The neodymium-yttrium aluminium garnet (Nd-YAG) laser can be used through a probe introduced through a flexible endoscope. ${ }^{9}$ It is possibly easier to use than a urological resectoscope. The problem with the Nd-YAG laser is that in 